\title{
A local limit theorem for Poisson binomial random variables
}

\author{
Tatpon Siripraparat ${ }^{\mathrm{a}}$, Kritsana Neammanee ${ }^{\mathrm{a}, \mathrm{b}, *}$ \\ a Department of Mathematics and Computer Science, Faculty of Science, Chulalongkorn University, \\ Bangkok 10330 Thailand \\ b Centre of Excellence in Mathematics, Commission on Higher Education, Bangkok 10400 Thailand
}

*Corresponding author, e-mail: Kritsana.N@chula.ac.th

Received 11 Jun 2020

Accepted 18 Nov 2020

ABSTRACT: We investigate the local limit theorem for Poisson binomial random variables $S_{n}:=\sum_{i=1}^{n} X_{i}$, where $X_{1}, X_{2}, \ldots, X_{n}$ are independent Bernoulli random variables. Using the idea from Neammanee [Int J Math Math Sci 5 (2005):717-728], we derive improved explicit bounds for the density of $S_{n}$.

KEYWORDS: Poisson binomial random variable, local limit theorem, normal density function

MSC2010: 60F05

\section{INTRODUCTION}

De Moivre and Laplace (see also [1]) established the local limit theorem for binomial case in 1754 . For sums of independent random variables, the local limit theorem began with the work of Esseen [2] and well understood by Petrov [3]. Since then, many researchers gave the local limit theorem in various versions (see [4-9] for examples). In this work, we are interested in the density of Poisson binomial random variables, i.e. $P\left(S_{n}=k\right)$, where $S_{n}:=\sum_{i=1}^{n} X_{i}$, and $X_{1}, X_{2}, \ldots, X_{n}$ are independent Bernoulli random variables with $p_{i}:=P\left(X_{i}=1\right), q_{i}:=P\left(X_{i}=0\right)$, and $p_{i}+q_{i}=1$ for $i=1,2, \ldots, n$.

Let $\mu:=\mathrm{E} S_{n}=\sum_{i=1}^{n} p_{i}$ and $\sigma^{2}:=\operatorname{Var} S_{n}=$ $\sum_{i=1}^{n} p_{i} q_{i}$. To approximate this probability, we use a local limit theorem that describes how $P\left(S_{n}=k\right)$ approaches the normal density, $\frac{1}{\sigma \sqrt{2 \pi}} \mathrm{e}^{-\frac{(k-\mu)^{2}}{2 \sigma^{2}}}$. The error bounds can be defined by

$$
\triangle_{n}=\sup _{k \in\{0,1, \ldots, n\}}\left|P\left(S_{n}=k\right)-\frac{1}{\sigma \sqrt{2 \pi}} \mathrm{e}^{-\frac{(k-\mu)^{2}}{2 \sigma^{2}}}\right| .
$$

In the case that $X_{1}, X_{2}, \ldots, X_{n}$ are independent identically distributed (i.i.d) integral-valued random variables with finite third moment. Petrov [3] showed that $\triangle_{n} \leqslant C_{1} / \sigma^{2}$, where $C_{1}>0$ is a constant.

In addition, if $S_{n}$ is a symmetric binomial with $P\left(X_{i}=0\right)=P\left(X_{i}=1\right)=1 / 2$, Petrov [3] (see also [10]) improved the rate of convergence from

$$
O\left(1 / \sigma^{2}\right) \text { to } O\left(1 / \sigma^{3}\right) \text {, i.e., }
$$

$$
\triangle_{n} \leqslant \frac{C_{2}}{n \sqrt{n}}
$$

where $C_{2}>0$ is a constant.

Explicit constants of the error bounds were not given until 1998 when Korolev and Zhukov [11] were able to give the rate of convergence with a constant of error bounds in the case of a continuous random variable. Shevtsova [12] later improved the result of Korolev and Zhukov and got a better constant in 2017 as in the following theorem.

Theorem 1 Let $X_{1}, X_{2}, \ldots, X_{n}$ be i.i.d absolutely continuous random variables with the density $p(x)$, satisfying the conditions: $\mathrm{E} X_{1}=0, \operatorname{Var} X_{1}=1$ and $\mathrm{E}\left|X_{1}\right|^{3}<\infty$. Assume that $A=\sup _{x} p(x)<\infty$. Let $S_{n}=\frac{1}{\sqrt{n}} \sum_{i=1}^{n} X_{i}$. Then, for all $n \geqslant 2$

$$
\begin{aligned}
\triangle_{n} \leqslant & \frac{1}{2 \pi} \frac{\mathrm{E}\left|X_{1}\right|^{3}}{\sqrt{n}}+1.2136 \frac{\mathrm{E}\left|X_{1}\right|^{3}}{\sqrt{n}} \mathrm{e}^{-0.0344 \frac{n}{\left(\mathrm{E}\left|X_{1}\right|^{3}\right)^{2}}} \\
& +A \sqrt{n}\left[1-\frac{0.0023}{A^{2}\left(\mathrm{E}\left|X_{1}\right|^{3}\right)^{2}}\left(1-\frac{0.0047}{\left(\mathrm{E}\left|X_{1}\right|^{3}\right)^{2}}\right)^{3}\right]^{n-2} .
\end{aligned}
$$

It should be noted that the constants of the error bounds were given in the case of continuous random variables. In 2018, Zolotukhin et al [13] gave the rate of convergence with a constant of error bounds in the case when $S_{n}$ is binomial with $P\left(X_{i}=1\right)=$ $p=1-P\left(X_{i}=0\right)$. They showed that if $n p q>1$, 
then

$$
\triangle_{n} \leqslant \min \left\{\frac{1}{\sqrt{2 \mathrm{enpq}}}, \frac{0.516}{n p q}\right\} .
$$

Observe that (1) gives a constant of the error bounds in the case when $X_{i}$ 's are identical. In this work, the condition of the random variables being identical will be relaxed.

Out results are as follows.

Theorem 2 Let $X_{1}, X_{2}, \ldots, X_{n}$ be independent Bernoulli random variables with $p_{i}:=P\left(X_{i}=1\right)$ and $q_{i}:=P\left(X_{i}=0\right)$, where $p_{i}+q_{i}=1$ for $i=1,2, \ldots, n$. Let $S_{n}=\sum_{i=1}^{n} X_{i}, \mathrm{E} S_{n}=\mu$ and $\operatorname{Var} S_{n}=\sigma^{2}$. Then for $\sigma^{2}>1$,

$$
\begin{aligned}
\triangle_{n} \leqslant & \frac{0.1194}{\sigma^{2}\left(1-\frac{3}{4 \sigma}\right)^{3}}+\frac{0.0749}{\sigma^{3}} \\
& +\frac{0.2107}{\sigma^{3}\left(1-\frac{3}{4 \sigma}\right)^{6}}+\left(\frac{0.4579}{\sqrt{\sigma}}+\frac{0.4725}{\sigma \sqrt{\sigma}}\right) \mathrm{e}^{-\frac{3}{2} \sigma} .
\end{aligned}
$$

For a special case when $X_{i}$ 's are identical, i.e., $P\left(X_{i}=1\right)=p=1-P\left(X_{i}=0\right), S_{n}$ is a binomial random variable with parameter $p$. Moreover, if $p=$ $1 / 2$, the rate of convergence can be improved from $O(1 / n)$ to $O(1 / n \sqrt{n})$ as in the following theorem.

Theorem 3 Let $S_{n} \sim B i(p)$. If $n p q>1$, then

$$
\begin{gathered}
\triangle_{n} \leqslant \frac{0.1194}{n p q\left(1-\frac{3}{4 \sqrt{n p q}}\right)^{3}}+\frac{0.0749}{n p q \sqrt{n p q}} \\
+\frac{0.2107}{n p q \sqrt{n p q}\left(1-\frac{3}{4 \sqrt{n p q}}\right)^{6}} \\
+\left(\frac{0.4579}{(n p q)^{1 / 4}}+\frac{0.4725}{(n p q)^{3 / 4}}\right) \mathrm{e}^{-\frac{3}{2} \sqrt{n p q}}
\end{gathered}
$$

Futhermore, if $p=1 / 2$ and $n>4$, then

$$
\begin{aligned}
\triangle_{n} \leqslant \frac{0.5992}{n \sqrt{n}}+ & \frac{3.3984}{n^{2}\left(1-\frac{3}{2 \sqrt{n}}\right)^{4}}+\frac{337.8048}{n^{3} \sqrt{n}\left(1-\frac{3}{2 \sqrt{n}}\right)^{8}} \\
& +\left(\frac{0.6476}{n^{1 / 4}}+\frac{1.3365}{n^{3 / 4}}\right) \mathrm{e}^{-\frac{3}{4} \sqrt{n}} .
\end{aligned}
$$

One can see that the constants in Theorem 2 and Theorem 3 can be expressed in terms of $n p q$ only. Moreover, the constants of the error bounds in (2) are smaller than those in (1) when $n$ is large enough. Theorem 3 can be applied to a random walk as in the following corollary.
Corollary 1 Let $S_{n}$ be a random walk, $P\left(X_{i}=1\right)=$ $p=1-P\left(X_{i}=-1\right)$ and

$$
\epsilon_{n}=\sup _{k \in\{0,1, \ldots, n\}}\left|P\left(S_{n}=k\right)-\frac{1}{\sqrt{n p q} \sqrt{2 \pi}} \mathrm{e}^{-\frac{\left(\frac{k+n}{2}-n p\right)^{2}}{2 n p q}}\right| .
$$

If $n p q>1$, then

$$
\begin{gathered}
\epsilon_{n} \leqslant \frac{0.1194}{n p q\left(1-\frac{3}{4 \sqrt{n p q}}\right)^{3}}+\frac{0.0749}{n p q \sqrt{n p q}} \\
+\frac{0.2107}{n p q \sqrt{n p q}\left(1-\frac{3}{4 \sqrt{n p q}}\right)^{6}} \\
+\left(\frac{0.4579}{(n p q)^{1 / 4}}+\frac{0.4725}{(n p q)^{3 / 4}}\right) \mathrm{e}^{-\frac{3}{2} \sqrt{n p q}} .
\end{gathered}
$$

In the case that $p=1 / 2$ and $n>4$, we have

$$
\begin{aligned}
\epsilon_{n} \leqslant \frac{0.5992}{n \sqrt{n}}+ & \frac{3.3984}{n^{2}\left(1-\frac{3}{2 \sqrt{n}}\right)^{4}}+\frac{337.8048}{n^{3} \sqrt{n}\left(1-\frac{3}{2 \sqrt{n}}\right)^{8}} \\
& +\left(\frac{0.6476}{n^{1 / 4}}+\frac{1.3365}{n^{3 / 4}}\right) \mathrm{e}^{-\frac{3}{4} \sqrt{n}} .
\end{aligned}
$$

\section{AUXILIARY RESULTS}

Let $X_{1}, X_{2}, \ldots, X_{n}$ be independent Bernoulli random variables with $p_{j}:=P\left(X_{j}=1\right)$ and $q_{j}:=P\left(X_{j}=\right.$ 0 ), where $p_{j}+q_{j}=1$ for $j=1,2, \ldots, n$. Let $S_{n}=$ $\sum_{i=1}^{n} X_{i}, \mu=\mathrm{E} S_{n}=\sum_{i=1}^{n} p_{i}$ and $\sigma^{2}=\operatorname{Var} S_{n}=$ $\sum_{i=1}^{n} p_{i} q_{i}$. Let $\psi_{1}, \psi_{2}, \ldots, \psi_{n}$ and $\psi$ be the characteristic functions of $X_{1}, X_{2}, \ldots, X_{n}$ and $S_{n}$, respectively. Then, for $j=1,2, \ldots, n$,

$$
\psi_{j}(t)=q_{j}+p_{j} \mathrm{e}^{\mathrm{i} t} \quad \text { and } \quad \psi(t)=\prod_{j=1}^{n}\left(q_{j}+p_{j} \mathrm{e}^{\mathrm{i} t}\right) .
$$

Note that

$$
\psi_{j}(t)=\left(q_{j}+p_{j} \cos (t)\right)+\mathrm{i} p_{j} \sin (t)=\rho_{j}(t) \mathrm{e}^{\mathrm{i} \theta_{j}(t)},
$$

where

$$
\begin{aligned}
& \theta_{j}(t)=\arctan \left(\frac{p_{j} \sin (t)}{q_{j}+p_{j} \cos (t)}\right), \\
& \rho_{j}(t)=\left|\psi_{j}(t)\right|=\left(1-4 p_{j} q_{j} \sin ^{2}\left(\frac{t}{2}\right)\right)^{1 / 2} .
\end{aligned}
$$

Hence, $\psi(t)=\rho(t) \mathrm{e}^{\mathrm{i} \theta(t)}$, where $\theta(t)=\sum_{j=1}^{n} \theta_{j}(t)$ $(\bmod 2 \pi)$, and $\rho(t)=\prod_{j=1}^{n} \rho_{j}(t)$.

Let $\alpha(t)=\theta(t)-\mu t$. In order to prove our main results, we use the ideas from Uspensky [14] and Neammanee [15] to obtain the following Lemma 1. 
Lemma 1 Let $t \in[0, \sqrt{3 / \sigma}]$. For $j=1,2, \ldots, n$, we have

(i) $\cos ((k-\mu) t-\alpha(t))=\cos ((k-\mu) t)+\triangle_{11}$, where $\left|\triangle_{11}\right| \leqslant \frac{0.1875 \sigma^{2} t^{3}}{\left(1-\frac{3}{4 \sigma}\right)^{3}}+\frac{0.0352 \sigma^{4} t^{6}}{\left(1-\frac{3}{4 \sigma}\right)^{6}}$.

(ii) If $p_{j}=q_{j}$, then

$$
\begin{aligned}
& \cos ((k-\mu) t-\alpha(t))=\cos ((k-\mu) t)+\triangle_{12}, \\
& \text { where }\left|\triangle_{12}\right| \leqslant \frac{0.0834 \sigma^{2} t^{5}}{\left(1-\frac{3}{4 \sigma}\right)^{4}}+\frac{0.007 \sigma^{4} t^{10}}{\left(1-\frac{3}{4 \sigma}\right)^{8}} .
\end{aligned}
$$

Proof: (i) Using the Taylor's expansion, we have for some $t_{0}, t_{1}, t_{2}, t_{3}$,

$$
\begin{aligned}
\cos (\alpha(t))= & 1-\frac{1}{2} \cos \left(t_{0}\right)(\alpha(t))^{2} \\
\sin (\alpha(t))= & \alpha(t)-\frac{1}{2} \sin \left(t_{1}\right)(\alpha(t))^{2} \\
\theta_{j}(t)= & \theta_{j}(0)+\theta_{j}^{(1)}(0) t+\frac{1}{2} \theta_{j}^{(2)}(0) t^{2} \\
& +\frac{1}{6} \theta_{j}^{(3)}\left(t_{2}\right) t^{3} \\
\theta_{j}(t)= & \theta_{j}(0)+\theta_{j}^{(1)}(0) t+\frac{1}{2} \theta_{j}^{(2)}(0) t^{2} \\
& +\frac{1}{6} \theta_{j}^{(3)}(0) t^{3}+\frac{1}{24} \theta_{j}^{(4)}\left(t_{3}\right) t^{4} .
\end{aligned}
$$

Note that

$$
\begin{gathered}
\theta_{j}^{(1)}(0)=p_{j}, \quad \theta_{j}^{(2)}(0)=0, \\
\theta_{j}^{(3)}(0)=p_{j} q_{j}\left(p_{j}-q_{j}\right)
\end{gathered}
$$

and for $t \in[0, \sqrt{3 / \sigma}]$

$$
\left|\theta_{j}^{(3)}(t)\right| \leqslant \frac{9 p_{j} q_{j}}{8\left(1-\frac{3}{4 \sigma}\right)^{3}}
$$

and

$$
\left|\theta_{j}^{(4)}(t)\right| \leqslant \frac{2 p_{j} q_{j} t}{\left(1-\frac{3}{4 \sigma}\right)^{4}},
$$

see page 722 [15] and also [14].

By (8), (10), and (11), we obtain

$$
|\alpha(t)| \leqslant \frac{1}{6} \frac{9 t^{3}}{8\left(1-\frac{3}{4 \sigma}\right)^{3}} \sum_{j=1}^{n} p_{j} q_{j}=\frac{0.1875 \sigma^{2} t^{3}}{\left(1-\frac{3}{4 \sigma}\right)^{3}}
$$

and

$$
\left|\alpha^{2}(t)\right| \leqslant \frac{0.0352 \sigma^{4} t^{6}}{\left(1-\frac{3}{4 \sigma}\right)^{6}} .
$$

From (6) and (7), we get

$$
\begin{aligned}
\cos ((k-\mu) t-\alpha(t)) \\
=\cos ((k-\mu) t) \cos (\alpha(t))+\sin ((k-\mu) t) \sin (\alpha(t)) \\
=\cos ((k-\mu) t)\left(1-\frac{1}{2} \cos \left(t_{0}\right) \alpha^{2}(t)\right) \\
\quad+\sin ((k-\mu) t)\left(\alpha(t)-\frac{1}{2} \sin \left(t_{1}\right) \alpha^{2}(t)\right) \\
=\cos ((k-\mu) t)+\triangle_{11},
\end{aligned}
$$

where

$$
\left|\triangle_{11}\right| \leqslant|\alpha(t)|+\alpha^{2}(t) .
$$

By (13), (14) and (16), we get

$$
\left|\triangle_{11}\right| \leqslant \frac{0.1875 \sigma^{2} t^{3}}{\left(1-\frac{3}{4 \sigma}\right)^{3}}+\frac{0.0352 \sigma^{4} t^{6}}{\left(1-\frac{3}{4 \sigma}\right)^{6}} .
$$

(ii) Since $p_{j}=q_{j}$, by (9), (10) and (12), we get

$$
|\alpha(t)| \leqslant \frac{0.0834 \sigma^{2} t^{5}}{\left(1-\frac{3}{4 \sigma}\right)^{4}},\left|\alpha^{2}(t)\right| \leqslant \frac{0.007 \sigma^{4} t^{10}}{\left(1-\frac{3}{4 \sigma}\right)^{8}} .
$$

The proof follows from (15), (16) and (17).

Lemma 2 For $j=1,2, \ldots, n$,

(i) $\rho_{j}(t) \leqslant \mathrm{e}^{-\frac{2}{\pi^{2}} p_{j} q_{j} t^{2}}$ for $t \in[0, \pi)$,

(ii) $\rho_{j}(t) \leqslant \mathrm{e}^{-\frac{1}{2} p_{j} q_{j} t^{2}+\frac{1}{24} p_{j} q_{j} t^{4}}$ for $t \in[0, \pi]$,

(iii) $\rho(t)$ is decreasing on $[0, \pi / 2]$,

(iv) $\left|\rho(t)-\mathrm{e}^{-\frac{1}{2} \sigma^{2} t^{2}}\right| \leqslant \frac{1}{16} \sigma^{2} t^{4} \mathrm{e}^{-\frac{1}{2} \sigma^{2} t^{2}}$ for $t \in\left[0, \sqrt{\frac{3}{\sigma}}\right]$.

Proof: See page 720-723 in [15], and also [14].

In the next lemma, we give the probability of integral-valued random variable.

Lemma 3 Let $X$ be any integral-valued random variable with finite variance and the characteristic function $\psi_{X}$. Then,

$P(X=k)=\frac{1}{\pi} \int_{0}^{\pi}\left|\psi_{X}(t)\right| \cos ((k-E(X)) t-\alpha(t)) \mathrm{d} t$,

where $\alpha(t)=\theta_{X}(t)-E(X) t$ and $\theta_{X}(t)$ is the argument of $\psi_{X}(t)$.

Proof: Note that, for each integer $k$,

$$
\begin{aligned}
\int_{-\pi}^{\pi} \mathrm{e}^{-\mathrm{i} k t} \psi_{X}(t) \mathrm{d} t & =\int_{-\pi}^{\pi} \mathrm{e}^{-\mathrm{i} k t}\left(\sum_{j=-\infty}^{\infty} P(X=j) \mathrm{e}^{\mathrm{i} j t}\right) \mathrm{d} t \\
& =\sum_{j=-\infty}^{\infty} P(X=j) \int_{-\pi}^{\pi} \mathrm{e}^{\mathrm{i}(j-k) t} \mathrm{~d} t \\
& =2 \pi P(X=k),
\end{aligned}
$$

and

$$
\begin{aligned}
\int_{-\pi}^{\pi} \mathrm{e}^{-\mathrm{i} k t} \psi_{X}(t) \mathrm{d} t & =\int_{-\pi}^{\pi} \mathrm{e}^{-\mathrm{i} k t}\left|\psi_{X}(t)\right| \mathrm{e}^{\mathrm{i} \theta_{X}(t)} \mathrm{d} t \\
& =\int_{-\pi}^{\pi}\left|\psi_{X}(t)\right| \mathrm{e}^{\mathrm{i}\left(\theta_{X}(t)-k t\right)} \mathrm{d} t \\
& =\int_{-\pi}^{\pi}\left|\psi_{X}(t)\right| \cos \left(\theta_{X}(t)-k t\right) \mathrm{d} t \\
& +i \int_{-\pi}^{\pi}\left|\psi_{X}(t)\right| \sin \left(\theta_{X}(t)-k t\right) \mathrm{d} t .
\end{aligned}
$$


From the above results and the fact that $P(X=k)$ is where real, we get

$$
P(X=k)=\frac{1}{2 \pi} \int_{-\pi}^{\pi}\left|\psi_{X}(t)\right| \cos \left(\theta_{X}(t)-k t\right) \mathrm{d} t .
$$

Observe that

$$
\begin{aligned}
& \psi_{X}(t)=\sum_{j=-\infty}^{\infty} P(X=j) \mathrm{e}^{\mathrm{i} j t} \\
& =\sum_{j=-\infty}^{\infty} P(X=j) \cos (j t)+\mathrm{i} \sum_{j=-\infty}^{\infty} P(X=j) \sin (j t) .
\end{aligned}
$$

Therefore,

$$
\begin{aligned}
\left|\psi_{X}(t)\right|= & {\left[\left(\sum_{j=-\infty}^{\infty} P(X=j) \cos (j t)\right)^{2}\right.} \\
& \left.+\left(\sum_{j=-\infty}^{\infty} P(X=j) \sin (j t)\right)^{2}\right]^{\frac{1}{2}}, \\
\theta_{X}(t)= & \arctan \left(\frac{\sum_{j=-\infty}^{\infty} P(X=j) \sin (j t)}{\sum_{j=-\infty}^{\infty} P(X=j) \cos (j t)}\right) .
\end{aligned}
$$

Since $\left|\psi_{X}(t)\right|$ is even and $\theta_{X}(t)$ is odd, $\left|\psi_{X}(t)\right| \cos \left(\theta_{X}(t)-k t\right)$ is even. Hence,

$$
\begin{aligned}
P(X=k) & =\frac{2}{2 \pi} \int_{0}^{\pi}\left|\psi_{X}(t)\right| \cos \left(\theta_{X}(t)-k t\right) \mathrm{d} t \\
= & \frac{1}{\pi} \int_{0}^{\pi}\left|\psi_{X}(t)\right| \cos ((k-E(X)) t-\alpha(t)) \mathrm{d} t .
\end{aligned}
$$

\section{PROOF OF THE MAIN RESULTS}

\section{Proof of Theorem 2}

Proof: By Lemma 3, we get

$$
\begin{array}{r}
P\left(S_{n}=k\right)=\frac{1}{\pi} \int_{0}^{\pi} \rho(t) \cos ((k-\mu) t-\alpha(t)) \mathrm{d} t \\
=\frac{1}{\pi} \int_{0}^{\sqrt{3 / \sigma}} \rho(t) \cos ((k-\mu) t-\alpha(t)) \mathrm{d} t+\triangle_{1},
\end{array}
$$

where

$$
\triangle_{1}=\frac{1}{\pi} \int_{\sqrt{3 / \sigma}}^{\pi} \rho(t) \cos ((k-\mu) t-\alpha(t)) \mathrm{d} t .
$$

Note that

$$
\left|\triangle_{1}\right| \leqslant \frac{1}{\pi} \int_{\sqrt{3 / \sigma}}^{\pi} \rho(t) \mathrm{d} t=\triangle_{11}+\triangle_{12},
$$

$$
\begin{aligned}
& \triangle_{11}=\frac{1}{\pi} \int_{\sqrt{\frac{3}{\sigma}}}^{\sqrt{\frac{3}{4 \sigma}} \pi} \rho(t) \mathrm{d} t, \\
& \triangle_{12}=\frac{1}{\pi} \int_{\sqrt{\frac{3}{4 \sigma}} \pi}^{\pi} \rho(t) \mathrm{d} t .
\end{aligned}
$$

By Lemma 2(ii) and Lemma 2(iii), we obtain

$$
\begin{aligned}
\left|\triangle_{11}\right| & \leqslant \frac{1}{\pi} \rho\left(\sqrt{\frac{3}{\sigma}}\right) \int_{\sqrt{\frac{3}{\sigma}}}^{\sqrt{\frac{3}{4 \sigma}} \pi} \mathrm{d} t \\
& \leqslant \frac{1}{\pi} \mathrm{e}^{\frac{9}{24}} \mathrm{e}^{-\frac{3}{2} \sigma} \int_{\sqrt{\frac{3}{\sigma}}}^{\sqrt{\frac{3}{4 \sigma}} \pi} \mathrm{d} t \\
& =\frac{1}{\pi} \mathrm{e}^{\frac{9}{24}} \mathrm{e}^{-\frac{3}{2} \sigma}\left(\sqrt{\frac{3}{4 \sigma}} \pi-\sqrt{\frac{3}{\sigma}}\right) \\
& \leqslant \frac{0.4579}{\sqrt{\sigma}} \mathrm{e}^{-\frac{3}{2} \sigma},
\end{aligned}
$$

and by Lemma 2(i), we get

$$
\begin{aligned}
\left|\triangle_{12}\right| & \leqslant \frac{1}{\pi} \int_{\sqrt{\frac{3}{4 \sigma}} \pi}^{\infty} \mathrm{e}^{-\frac{2}{\pi^{2}} \sigma^{2} t^{2}} \mathrm{~d} t \\
& =\frac{1}{\pi} \int_{\sqrt{3 \sigma / 2}}^{\infty} \frac{\pi}{\sqrt{2} \sigma} \mathrm{e}^{-t^{2}} \mathrm{~d} t \\
& \leqslant \frac{1}{\sigma} \frac{\sqrt{2}}{\sqrt{6 \sigma}} \int_{\sqrt{3 \sigma / 2}}^{\infty} t \mathrm{e}^{-t^{2}} \mathrm{~d} t \\
& =\frac{1}{\sigma \sqrt{3 \sigma}}\left(\frac{\mathrm{e}^{-\frac{3}{2} \sigma}}{2}\right) \\
& \leqslant \frac{0.2887}{\sigma \sqrt{\sigma}} \mathrm{e}^{-\frac{3}{2} \sigma} .
\end{aligned}
$$

Hence, by (18)-(21),

$P\left(S_{n}=k\right)=\frac{1}{\pi} \int_{0}^{\sqrt{3 / \sigma}} \rho(t) \cos ((k-\mu) t-\alpha(t)) \mathrm{d} t+\triangle_{1}$,

where

$\left|\triangle_{1}\right| \leqslant\left|\triangle_{11}\right|+\left|\triangle_{12}\right| \leqslant \frac{0.4579}{\sqrt{\sigma}} \mathrm{e}^{-\frac{3}{2} \sigma}+\frac{0.2887}{\sigma \sqrt{\sigma}} \mathrm{e}^{-\frac{3}{2} \sigma}$.

By Lemma 2(iv), we obtain

$$
\begin{aligned}
& \frac{1}{\pi} \int_{0}^{\sqrt{3 / \sigma}} \rho(t) \cos ((k-\mu) t-\alpha(t)) \mathrm{d} t \\
& \quad=\frac{1}{\pi} \int_{0}^{\sqrt{3 / \sigma}} \mathrm{e}^{-\frac{1}{2} \sigma^{2} t^{2}} \cos ((k-\mu) t-\alpha(t)) \mathrm{d} t+\triangle_{2}
\end{aligned}
$$


where

$$
\begin{aligned}
\left|\triangle_{2}\right| & \leqslant \frac{\sigma^{2}}{16 \pi} \int_{0}^{\sqrt{3 / \sigma}} t^{4} \mathrm{e}^{-\frac{1}{2} \sigma^{2} t^{2}} \mathrm{~d} t \\
& \leqslant \frac{\sigma^{2}}{16 \pi} \int_{0}^{\infty} t^{4} \mathrm{e}^{-\frac{1}{2} \sigma^{2} t^{2}} \mathrm{~d} t \\
& =\frac{\sigma^{2}}{16 \pi}\left(\frac{3 \sqrt{\pi}}{\sqrt{2} \sigma^{5}}\right) \leqslant \frac{0.0749}{\sigma^{3}} .
\end{aligned}
$$

By Lemma 1(i), we get

$$
\begin{aligned}
& \frac{1}{\pi} \int_{0}^{\sqrt{3 / \sigma}} \mathrm{e}^{-\frac{1}{2} \sigma^{2} t^{2}} \cos ((k-\mu) t-\alpha(t)) \mathrm{d} t \\
& \quad=\frac{1}{\pi} \int_{0}^{\sqrt{3 / \sigma}} \mathrm{e}^{-\frac{1}{2} \sigma^{2} t^{2}} \cos ((k-\mu) t) \mathrm{d} t+\triangle_{3},
\end{aligned}
$$

where

$$
\begin{gathered}
\left|\triangle_{3}\right| \leqslant \frac{1}{\pi} \int_{0}^{\sqrt{\frac{3}{\sigma}}}\left(\frac{0.1875 \sigma^{2} t^{3}}{\left(1-\frac{3}{4 \sigma}\right)^{3}}+\frac{0.0352 \sigma^{4} t^{6}}{\left(1-\frac{3}{4 \sigma}\right)^{6}}\right) \mathrm{e}^{-\frac{1}{2} \sigma^{2} t^{2}} \mathrm{~d} t \\
\leqslant \frac{0.1875 \sigma^{2}}{\pi\left(1-\frac{3}{4 \sigma}\right)^{3}}\left(\frac{2}{\sigma^{4}}\right)+\frac{0.0352 \sigma^{4}}{\pi\left(1-\frac{3}{4 \sigma}\right)^{6}}\left(\frac{15 \sqrt{\pi}}{\sqrt{2} \sigma^{7}}\right) \\
\leqslant \frac{0.1194}{\sigma^{2}\left(1-\frac{3}{4 \sigma}\right)^{3}}+\frac{0.2107}{\sigma^{3}\left(1-\frac{3}{4 \sigma}\right)^{6}}
\end{gathered}
$$

From (22)-(25),

$P\left(S_{n}=k\right)=\frac{1}{\pi} \int_{0}^{\sqrt{3 / \sigma}} \mathrm{e}^{-\frac{1}{2} \sigma^{2} t^{2}} \cos ((k-\mu) t) \mathrm{d} t+\triangle_{4}$,

where

$$
\begin{aligned}
\left|\triangle_{4}\right| \leqslant & \left|\triangle_{1}\right|+\left|\triangle_{2}\right|+\left|\triangle_{3}\right| \\
\leqslant & \frac{0.4579}{\sqrt{\sigma}} \mathrm{e}^{-\frac{3}{2} \sigma}+\frac{0.2887}{\sigma \sqrt{\sigma}} \mathrm{e}^{-\frac{3}{2} \sigma}+\frac{0.0749}{\sigma^{3}} \\
& +\frac{0.1194}{\sigma^{2}\left(1-\frac{3}{4 \sigma}\right)^{3}}+\frac{0.2107}{\sigma^{3}\left(1-\frac{3}{4 \sigma}\right)^{6}} .
\end{aligned}
$$

It is known that

$$
\int_{0}^{\infty} \mathrm{e}^{-a t^{2}} \cos (b t) \mathrm{d} t=\frac{1}{2} \sqrt{\frac{\pi}{a}} \mathrm{e}^{-\frac{b^{2}}{4 a}} \text { for } a>0 .
$$

We thus obtain

$$
\begin{aligned}
\frac{1}{\pi} \int_{0}^{\sqrt{3 / \sigma}} & \mathrm{e}^{-\frac{1}{2} \sigma^{2} t^{2}} \cos ((k-\mu) t) \mathrm{d} t \\
& =\frac{1}{\pi} \int_{0}^{\infty} \mathrm{e}^{-\frac{1}{2} \sigma^{2} t^{2}} \cos ((k-\mu) t) \mathrm{d} t+\triangle_{5} \\
& =\frac{1}{\sigma \sqrt{2 \pi}} \mathrm{e}^{-\frac{(k-\mu)^{2}}{2 \sigma^{2}}}+\triangle_{5}
\end{aligned}
$$

where

$$
\begin{aligned}
\left|\triangle_{5}\right| & \leqslant \frac{1}{\pi} \int_{\sqrt{3 / \sigma}}^{\infty} \mathrm{e}^{-\frac{1}{2} \sigma^{2} t^{2}} \mathrm{~d} t \\
& \leqslant \frac{1}{\pi} \sqrt{\frac{\sigma}{3}} \int_{\sqrt{3 / \sigma}}^{\infty} t \mathrm{e}^{-\frac{1}{2} \sigma^{2} t^{2}} \mathrm{~d} t \\
& =\frac{1}{\pi} \sqrt{\frac{\sigma}{3}}\left(\frac{\mathrm{e}^{-\frac{3}{2} \sigma}}{\sigma^{2}}\right) \leqslant \frac{0.1838}{\sigma \sqrt{\sigma}} \mathrm{e}^{-\frac{3}{2} \sigma} .
\end{aligned}
$$

From (26)-(28), we can conclude that

$$
P\left(S_{n}=k\right)=\frac{1}{\sigma \sqrt{2 \pi}} \mathrm{e}^{-\frac{(k-\mu)^{2}}{2 \sigma^{2}}}+\triangle_{6},
$$

where

$$
\begin{aligned}
\left|\triangle_{6}\right| \leqslant & \left|\triangle_{4}\right|+\left|\triangle_{5}\right| \\
\leqslant & \frac{0.4579}{\sqrt{\sigma}} \mathrm{e}^{-\frac{3}{2} \sigma}+\frac{0.2887}{\sigma \sqrt{\sigma}} \mathrm{e}^{-\frac{3}{2} \sigma}+\frac{0.0749}{\sigma^{3}} \\
& +\frac{0.1194}{\sigma^{2}\left(1-\frac{3}{4 \sigma}\right)^{3}}+\frac{0.2107}{\sigma^{3}\left(1-\frac{3}{4 \sigma}\right)^{6}}+\frac{0.1838}{\sigma \sqrt{\sigma}} \mathrm{e}^{-\frac{3}{2} \sigma} \\
= & \frac{0.1194}{\sigma^{2}\left(1-\frac{3}{4 \sigma}\right)^{3}}+\frac{0.0749}{\sigma^{3}}+\frac{0.2107}{\sigma^{3}\left(1-\frac{3}{4 \sigma}\right)^{6}} \\
& +\left(\frac{0.4579}{\sqrt{\sigma}}+\frac{0.4725}{\sigma \sqrt{\sigma}}\right) \mathrm{e}^{-\frac{3}{2} \sigma} .
\end{aligned}
$$

\section{Proof of Theorem 3}

Proof: Since $S_{n} \sim B i(p), \sigma^{2}=n p q$. By Theorem 2, we get (2). By Lemma 1(ii), we get

$$
\begin{aligned}
& \frac{1}{\pi} \int_{0}^{\sqrt{3 / \sigma}} \mathrm{e}^{-\frac{1}{2} \sigma^{2} t^{2}} \cos ((k-\mu) t-\alpha(t)) \mathrm{d} t \\
& =\frac{1}{\pi} \int_{0}^{\sqrt{3 / \sigma}} \mathrm{e}^{-\frac{1}{2} \sigma^{2} t^{2}} \cos ((k-\mu) t) \mathrm{d} t+\Delta_{7},
\end{aligned}
$$

where

$$
\begin{aligned}
\left|\triangle_{7}\right| & \leqslant \frac{1}{\pi} \int_{0}^{\sqrt{\frac{3}{\sigma}}}\left(\frac{0.0834 \sigma^{2} t^{5}}{\left(1-\frac{3}{4 \sigma}\right)^{4}}+\frac{0.007 \sigma^{4} t^{10}}{\left(1-\frac{3}{4 \sigma}\right)^{8}}\right) \mathrm{e}^{-\frac{1}{2} \sigma^{2} t^{2}} \mathrm{~d} t \\
& \leqslant \frac{0.0834 \sigma^{2}}{\pi\left(1-\frac{3}{4 \sigma}\right)^{4}}\left(\frac{8}{\sigma^{6}}\right)+\frac{0.007 \sigma^{4}}{\pi\left(1-\frac{3}{4 \sigma}\right)^{8}}\left(\frac{945 \sqrt{\pi}}{\sqrt{2} \sigma^{11}}\right) \\
& \leqslant \frac{0.2124}{\sigma^{4}\left(1-\frac{3}{4 \sigma}\right)^{4}}+\frac{2.6391}{\sigma^{7}\left(1-\frac{3}{4 \sigma}\right)^{8}} .
\end{aligned}
$$

By (22)-(23), (27)-(28) and (29)-(30), we can conclude that

$$
P\left(S_{n}=k\right)=\frac{1}{\sigma \sqrt{2 \pi}} \mathrm{e}^{-\frac{(k-\mu)^{2}}{2 \sigma^{2}}}+\triangle_{8},
$$


where

$$
\begin{aligned}
\left|\triangle_{8}\right| \leqslant & \left|\triangle_{1}\right|+\left|\triangle_{2}\right|+\left|\triangle_{5}\right|+\left|\triangle_{7}\right| \\
\leqslant & \frac{0.4579}{\sqrt{\sigma}} \mathrm{e}^{-\frac{3}{2} \sigma}+\frac{0.2887}{\sigma \sqrt{\sigma}} \mathrm{e}^{-\frac{3}{2} \sigma}+\frac{0.0749}{\sigma^{3}} \\
& +\frac{0.1838}{\sigma \sqrt{\sigma}} \mathrm{e}^{-\frac{3}{2} \sigma}+\frac{0.2124}{\sigma^{4}\left(1-\frac{3}{4 \sigma}\right)^{4}}+\frac{2.6391}{\sigma^{7}\left(1-\frac{3}{4 \sigma}\right)^{8}} \\
= & \frac{0.0749}{\sigma^{3}}+\frac{0.2124}{\sigma^{4}\left(1-\frac{3}{4 \sigma}\right)^{4}}+\frac{2.6391}{\sigma^{7}\left(1-\frac{3}{4 \sigma}\right)^{8}} \\
& +\left(\frac{0.4579}{\sqrt{\sigma}}+\frac{0.4725}{\sigma \sqrt{\sigma}}\right) \mathrm{e}^{-\frac{3}{2} \sigma} .
\end{aligned}
$$

Hence, by $\sigma^{2}=n / 4$, we have (3).

\section{Proof of Corollary 1}

Proof: Let $Y_{i}=\left(X_{i}+1\right) / 2$. Then, $\mathrm{E}\left(\sum_{i=1}^{n} Y_{i}\right)=n p$, $\operatorname{Var}\left(\sum_{i=1}^{n} Y_{i}\right)=n p q$ and

$$
\begin{aligned}
P\left(S_{n}=k\right) & =P\left(\sum_{i=1}^{n} X_{i}=k\right) \\
& =P\left(\sum_{i=1}^{n}\left(\frac{X_{i}+1}{2}\right)=\frac{k+n}{2}\right) \\
& =P\left(\sum_{i=1}^{n} Y_{i}=\frac{k+n}{2}\right) .
\end{aligned}
$$

By Theorem 2, we obtain (4). Moreover, if $p=1 / 2$ and $n>4$, we get (5).

Acknowledgements: The authors are thankful for financial support by Development and Promotion of Science and Technology Talents Project (DPST). We also would like to thank the reviewers for their valuable comments and suggestions.

\section{REFERENCES}

1. McDonald DR (2005) The local limit theorem: A historical perspective. J Iran Stat Soc 4, 73-86.
2. Esseen CG (1945) Fourier analysis of distribution functions. A mathematical analysis of the LaplaceGaussian law. Acta Math 77, 1-125.

3. Petrov VV (1975) Sums of Independent Random Variables, Springer, Berlin.

4. Barbour AD, Röllin A, Ross N (2019) Error bounds in local limit theorems using Stein's method. Bernoulli 25, 1076-1104.

5. Barbour AD, Röllin A, Ross N (2020) Local limit theorems for occupancy models. arXiv:1908.00251.

6. Röllin AD, Ross N (2015) Local limit theorems via Landau-Kolmogorov inequalities. Bernoulli 21, 851-880.

7. McDonald DR (1979) On local limit theorem for integer valued random variables. Theor Probab Appl 24, 613-619.

8. Hwang HK, Janson S (2008) Local limit theorems for finite and infinite urn models. Ann Probab 36, 992-1022.

9. Penrose MD, Peres Y (2011) Local central limit theorems in stochastic geometry. Electron $J$ Probab 16, 2509-2544.

10. Giuliano R, Weber M (2017) Approximate local limit theorems with effective rate and application to random walks in random scenery. Bernoulli 23, 3268-3310.

11. Korolev VY, Zhukov YV (1998) On the rate of convergence in the local central limit theorem for densities. J Math Sci 91, 2931-2941.

12. Shevtsova IG (2017) On convergence rate in the local limit theorem for densities under various moment conditions. J Math Sci 221, 588-608.

13. Zolotukhin A, Nagaev S, Chebotarev V (2018) On a bound of the absolute constant in the Berry-Esseen inequality for i.i.d. Bernoulli random variables. Mod Stoch Theory Appl 5, 385-410.

14. Upensky JV (1937) Introduction to Mathematical Probability, McGraw-Hill, New York.

15. Neammanee K (2005) A refinement of normal approximation to Poisson binomial. Int J Math Math Sci 5, 717-728. 